\title{
Evaluación de la atención a usuarios con enfermedad crónica cardiovascular en Centros de Salud Familiar
}

\author{
María Elena Lagos ${ }^{a}$, Alide Salazar ${ }^{b}$, Luis Humberto Luengo ${ }^{c}$. \\ a Enfermera, Magister en Enfermería. Doctor@ en Enfermería Universidad de Concepción, Chile. \\ b Enfermera-Matrona. Doctora en Enfermería. Profesor Asociado, Facultad de Enfermería, \\ Universidad de Concepción, Chile. \\ $c$ \\ Profesor de Matemática y Física. Magister en Estadística Aplicada. \\ Universidad de Concepción, Chile. \\ Financiamiento: Convenio de Desempeño UCO1201 de la Universidad de Concepción para el \\ desarrollo de Tesis de Magíster.
}

Antecedentes: El control de las enfermedades cardiovasculares es un reto para los sistemas de salud. Objetivo: Determinar si la atención proporcionada por los equipos de salud a usuarios/as con enfermedades cardiovasculares crónicas es congruente con el Modelo de Cuidados Crónicos y explorar su efectividad a través de indicadores de compensación del programa de salud cardiovascular.

Métodos: Se empleó un diseño descriptivo, transversal. Participaron los equipos de salud (9) que otorgan atención a usuarios crónicos (diabéticos e hipertensos) en Centros de Salud Familiar de un Servicio de Salud del Centro Sur de Chile. Se utilizó el instrumento "Evaluación de la atención de enfermedades crónicas" (ACIC) versión 3.5 adaptada al castellano Chileno. Se analizaron los Indicadores de compensación para hipertensión arterial y diabetes mellitus tipo 2 del programa cardiovascular obtenidos del Resumen
Estadístico Mensual de cada centro de salud.

Resultados: La atención otorgada a usuarios/as con enfermedad crónica fue evaluada como apoyo básico a las enfermedades crónicas. La sección mejor evaluada fue "Organización del Sistema de atención de salud". Las dimensiones con puntajes más bajos fueron "Integración de los componentes del modelo" y "Apoyo a la toma de decisiones". Se observó solo una tendencia a correlación positiva entre la evaluación de la atención otorgada a usuarios/as y los indicadores de compensación para diabetes mellitus tipo 2, no así para hipertensión.

Conclusión: La "Organización del Sistema de atención de salud" fue la sección mejor evaluada del Modelo de Cuidados Crónicos en 9 Centros de Atención Familiar. No se encontró relación estadísticamente significativa con los indicadores de compensación para diabetes mellitus tipo 2 e hipertensión arterial.

\section{Correspondencia:}

Alide Salazar M.

Facultad de Enfermería, Universidad de Concepción.

Barrio Universitario s/n Concepción, Chile.

alisalaz@udec.cl. 


\section{Evaluation of care provided to users suffering from chronic cardiovascular disease at Family Health Centers}

Background: Control of cardiovascular disease remains a challenge for health care systems.

Objective: To determine whether care provided by health teams to users suffering from chronic cardiovascular diseases is consistent with the Chronic Care Model, and to explore it's effectiveness using compensation indicator from the cardiovascular health programs.

Methods: A descriptive cross-sectional design was used. 9 health teams providing assistance to chronic users (diabetes and hypertension) in Family Health Centers in southern-central Chile were included. We used ACIC version 3.5 adapted to Chilean Spanish to evaluate quality of chronic care. Indicators of compensation for hypertension and diabetes mellitus type 2 defined by national cardiovascular programs were obtained from the monthly statistical summary of each health center.
Results: "Organization of the Healthcare Delivery System" was the best evaluated dimension. The lower scores corresponded to "Integration of Chronic Care Model Components" and "Decision Support". Only a tendency for a positive correlation wasfound between care given to users suffering from chronic disease and compensation indicators for type 2 diabetes mellitus. No such tendency was observed for hypertension.

Conclusion: "Organization of the Healthcare Delivery System" was the best evaluated dimension of the Chronic Care Model in 9 Health temas delivering care to chronic users in Chile The relation between chronic care dimensions and compensation indicators for type 2 diabetes mellitus and hypertension was not satisfactory.

Key words: Chronic disease, health care delivery, patient care team. 
Introducción: El control insatisfactorio de la presión arterial (PA), de acuerdo a lo recomendado por las guías clínicas $(<140 / 90 \mathrm{mmHg})$, es un problema persistente en la población hipertensa a nivel mundial ${ }^{1,2}$, reportándose en Chile un control satisfactorio de la PA que alcanza a un $65 \%$ de la población hipertensa ${ }^{3}$. En el caso de la diabetes mellitus tipo 2 , las encuestas poblacionales en muchos países indican que más del 50\% de los pacientes no alcanzan el control glicémico ${ }^{4}$, reportándose en Chile un control satisfactorio ( $\mathrm{HbAl} 1 \mathrm{c}<7 \%$ ) del $42 \%$ de los diabéticos en control $^{3}$. En la Región del Bío - Bío, ubicada en el centro-sur chileno, estas cifras se encuentran bajo el promedio nacional, con un $61 \%$ para hipertensión y un $41 \%$ para diabetes mellitus tipo $2^{3}$.

Se ha hecho referencia a numerosos factores como responsables de estos deficientes resultados, que incluyen deficiencias en la organización, seguimiento y tratamiento de la atención de enfermedades crónicas. Ello se debería fundamentalmente a 3 problemas 5 : a) los pacientes pueden estar largos períodos sin que nadie se preocupe por sus condiciones, lo que disminuye la adhesión a las terapias y tratamientos, resultando en mala salud a un costo muy elevado para las familias, los gobiernos y la sociedad ${ }^{6}$; b) el sistema sanitario y los modelos de salud tradicionales están preparados para la atención reactiva de problemas agudos, reagudizaciones y complicaciones, no para la atención proactiva de los enfermos crónicos ${ }^{5,6-9}$, y c) los profesionales de atención de salud continúan otorgando cuidados de forma rutinaria; la formación, educación y conocimientos de los expertos de la salud de hoy día no son los adecuados para la atención a los enfermos crónicos ${ }^{6-9}$. Por otra parte, las intervenciones tradicionales (consultas y controles, educación, medicamentos) no han logrado resolver estos problemas, y se prevé que la atención no mejorará con agregar más estrés al sistema actual ${ }^{9}$. Es así como la forma en que se otorgan los cuidados actualmente conlleva a una identificación inadecuada de los usuarios en la población, escasa coordinación entre los servicios que los atienden, evaluación solo de resultados en la población y deficiente calidad en la atención otorgada ${ }^{7-11}$.

Rediseñar y reestructurar la atención de salud, ha sido una propuesta de diferentes organizaciones a nivel mundial. Uno de los modelos de gestión de cuidados de enfermedades crónicas más ampliamente utilizado corresponde al Modelo de Cuidados Crónicos (MCC) de Wagner ${ }^{12,13}$. En Chile, su implementación en establecimientos de atención primaria resulta de una propuesta Ministerial plasmada en los objetivos estratégicos 2011-2020. Este Modelo es un sistema efectivo de entrega de cuidado para la mejora de la calidad, que busca lograr los mejores resultados, controlar los costos y dar énfasis a investigar la evidencia. Sus metas son mejorar resultados clínicos, de satisfacción y costos, alcanzar la seguridad y lograr relaciones continuas a través de interacciones entre equipos preparados y proactivos y personas informadas y activas ${ }^{5,8,10,11}$.

Sin embargo, en Chile a la fecha se desconoce en qué medida ha sido implementado en la atención primaria y si la atención de los usuarios crónicos se relaciona con los resultados de compensación propuestos por el Ministerio de Salud. ${ }^{10,11}$

El objetivo del presente estudio fue determinar si la atención proporcionada por los equipos de un Servicio de Salud a usuarios/as crónicos con hipertensión arterial y/o diabetes mellitus tipo 2, en el nivel primario de atención, es congruente con el Modelo de Cuidados Crónicos (MCC) y explorar la relación entre la evaluación de la atención otorgada y los indicadores de compensación del programa de salud cardiovascular.

Los resultados de este estudio permitirán, en primer término, identificar áreas de mejoramiento y proponer estrategias, en caso de ser necesario, orientando la toma de decisiones por parte de las autoridades para el mejoramiento de la calidad de la prestación de salud.

\section{Métodos}

Se efectuó una Investigación descriptiva y transversal. En el Servicio de Salud estudiado, ubicado en la Región del Bío Bío en Chile, existen 10 Centros de Salud de dependencia municipal y se invitó a participar a todos los equipos de salud cardiovascular (10 equipos) que realizaran atención a usuarios crónicos cardiovasculares en establecimientos de nivel primario.

Criterios de inclusión. Personal del equipo de salud que realizara atención de salud a usuarios crónicos del programa cardiovascular en el nivel primario; autorización por parte del establecimiento de salud para participar en la investigación y haber otorgado su consentimiento informado.

Criterios de exclusión. Encontrase con licencia médica durante el periodo de recolección de datos y que el establecimiento de salud no dispusiera de la información relativa a los Indicadores de compensación para hipertensión arterial y diabetes mellitus tipo 2 .

Todos los individuos que cumplieron con los criterios de inclusión y aceptaron participar en la investigación fueron incluidos (no se efectuó muestreo) (Tabla 1). Cabe men- 
Tabla 1. Personal del equipo de salud que realizara atención de salud a usuarios del PSCV y participantes del estudio por Centro de salud

$\begin{array}{ccc}\text { CESFAM } & \begin{array}{c}\text { Total integrantes del equipo } \\ \text { que realiza atención a } \\ \text { usuarios del PSCV }\end{array} & \begin{array}{c}\text { Participantes del } \\ \text { estudio }\end{array} \\ \text { A } & 10 & \\ \text { B } & 25 & 5 \\ \text { C } & 20 & 11 \\ \text { D } & 15 & 13 \\ \text { E } & 20 & 8 \\ \text { F } & 25 & 10 \\ \text { G } & 25 & 11 \\ \text { H } & 15 & 15 \\ \text { I } & 15 & 15 \\ \text { J } & 5 & 5 \\ & 175 & 2 \\ & & 96\end{array}$

cionar que en uno de los Centros de salud sólo 2 miembros del equipo accedieron a participar, por lo que se consideró no incorporar este Centro, ya que estudios previos han mostrado que el número óptimo de participantes debe ser de al menos tres ${ }^{14}$.

Mediciones. La evaluación de la atención de enfermedades crónicas usó ACIC versión 3.5: La adaptación al castellano Chileno del "Assessment of Chronic Illness Care 3.5", fue realizada por Arteaga et al** en Centros de Atención Primaria de la Región Metropolitana en Chile. Consta de 7 sub-escalas o secciones: sección 1: Organización del
Sistema de Atención de Salud, sección 2: Vínculos con la Comunidad, sección 3: Nivel de atención de pacientes, sección 3a: Apoyo al auto-cuidado, sección 3b: Apoyo a la Toma de Decisiones, sección 3c: Diseño del Sistema de Atención, sección 3d: Sistemas de Información Clínica, Integración de los componentes del Modelo de Atención de Enfermedad Crónicas. La interpretación de los puntajes, en cualquier sección o a nivel global es: entre " 0 " y "2", apoyo limitado a la atención de enfermedades crónicas; entre "3" y "5", apoyo básico a la atención de enfermedades crónicas; entre "6" y "8" apoyo razonablemente bueno a la atención de enfermedades crónicas y entre "9" y "11" atención de enfermedades crónicas totalmente desarrollada.

Para evaluar la efectividad del modelo de atención se usaron indicadores de compensación para hipertensión arterial y diabetes mellitus tipo 2 del programa cardiovascular: contenidos en el Resumen Estadístico Mensual (REM) de cada establecimiento de salud. Estos indicadores corresponden al porcentaje de usuarios/as con hipertensión arterial compensados ( $\mathrm{PA}<140 / 90 \mathrm{mmHg}$ ) y al porcentaje de usuarios con diabetes mellitus tipo 2 compensados (HbAlc $<7 \mathrm{mg} / \mathrm{dl})$.

Recolección de datos. Se realizó con previa autorización del Servicio de Salud, Direcciones de Salud Municipal y Centros de Salud Familiar, durante el segundo semestre del año 2013. Los cuestionarios fueron respondidos de forma individual por los miembros del equipo de salud en sus

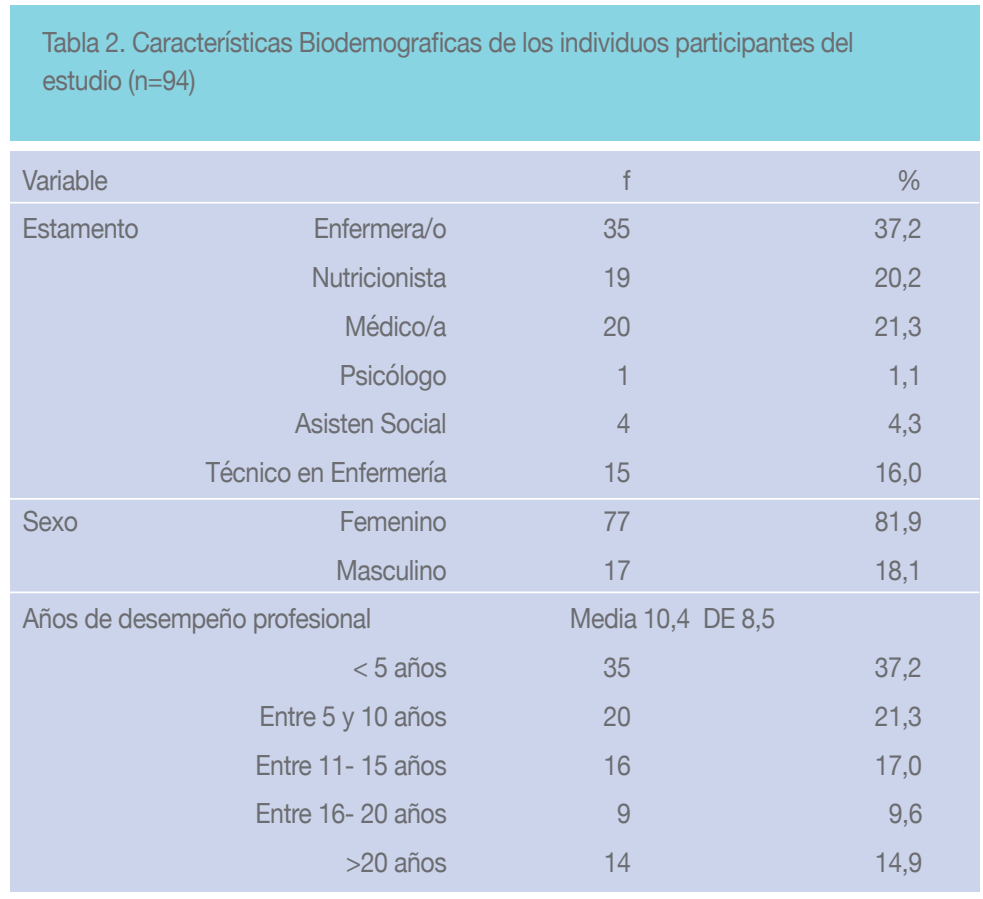

*Arteaga et al. Validación y aplicación del instrumento ACIC (Assessment of Chronic Illness Care) en centros de atención primaria de la Región Metropolitana (Chile). Fondo Nacional de Investigación y Desarrollo en Salud (FONIS) N SA07I20014. 
respectivos lugares de trabajo, previa entrevista para dar a conocer los objetivos del estudio y solicitar su consentimiento informado. Cuando no se encontró a todo el equipo se procedió a solicitar una segunda visita hasta completar la información necesaria.

La información de los indicadores de compensación del programa cardiovascular se solicitó a cada establecimiento.

Consideraciones éticas. El estudio fue aprobado por los Comités de Ética de la Facultad de Medicina de la Universidad de Concepción, según informe emitido 11/3/2013, y del Servicio de Salud, según consta en Oficio N²076. Posteriormente se solicitó autorización a las autoridades de las cuatro Direcciones de Salud Municipal, así como a los respectivos Directores/as de los Centros de Salud. Se tuvieron en cuenta los requisitos éticos que debe contemplar toda investigación científica, según Ezequiel Emmanuel ${ }^{15}$.

Procesamiento y análisis de los datos. Se realizó análisis descriptivo con tablas de frecuencias (variables cualitativas), medidas de tendencia central y dispersión (variables cuantitativas) con verificación de normalidad para la variable evaluación de la atención de enfermedades cróni- cas (prueba Kolmogorov-Smirnov). Además, se calculó el coeficiente de correlación de Pearson entre la evaluación de la atención de enfermedades crónicas y los indicadores de compensación para hipertensión arterial y diabetes mellitus tipo 2 del programa cardiovascular, de cada centro, acompañando con un gráfico de dispersión. El análisis se realizó con el software SPSS versión 12.0.

\section{Resultados}

La Tabla 2 muestra que la mayor proporción de encuestados correspondió a Enfermeros(as), Médicos(as) y Nutricionistas, observándose un predominio del sexo femenino. La duración del desempeño profesional promedio fue 10,4 años (DE 8,5). La variable "evaluación de la atención de enfermedades crónicas", considerando todos funcionarios ( $\mathrm{n}=94)$, tuvo distribución normal $(\mathrm{K}-\mathrm{S} \mathrm{p}=0,20)$. La Tabla 3 muestra que la atención otorgada fue evaluada como apoyo básico a enfermedades crónicas, con un promedio 5,9 (DE 1,5). La sección mejor evaluada fue "Organización del Sistema de Atención de Salud" con un promedio de 7,2 (DE 1,5), mientras que las secciones con más bajos puntajes fueron "Apoyo a la Toma de Decisiones": 4,8 (DE 2) e "Integración de los Componentes del Modelo": con un 4,7 (DE 1,9). Esta evaluación fue concordante en todos los equipos de salud.

\begin{tabular}{|c|c|c|c|c|c|c|c|c|c|c|c|c|c|c|c|}
\hline \multicolumn{16}{|c|}{ Secciones del instrumento ACIC 3.5 Evaluación de la atención proporcionada a usuarios/as con enfermedad crónica. } \\
\hline \multirow{2}{*}{$\begin{array}{l}\text { Equipo de } \\
\text { Salud Familiar } \\
\text { A }(n=5)\end{array}$} & \multicolumn{2}{|c|}{$\begin{array}{c}\text { Organización del } \\
\text { Sistema de atención } \\
\text { de salud }\end{array}$} & \multicolumn{2}{|c|}{$\begin{array}{l}\text { Vínculos con } \\
\text { la comunidad }\end{array}$} & \multicolumn{2}{|c|}{$\begin{array}{c}\text { Apoyo al } \\
\text { autocuidado }\end{array}$} & \multicolumn{2}{|c|}{$\begin{array}{c}\text { Apoyo a la toma } \\
\text { de decisiones }\end{array}$} & $\begin{array}{l}\text { Diseño del } \\
\text { sistema de } \\
\text { atención }\end{array}$ & \multicolumn{2}{|c|}{$\begin{array}{l}\text { Sistema de } \\
\text { información } \\
\text { Clínica }\end{array}$} & \multicolumn{2}{|c|}{$\begin{array}{l}\text { Integración de } \\
\text { los componen- } \\
\text { tes del modelo }\end{array}$} & \multicolumn{2}{|c|}{$\begin{array}{l}\text { ACIC } \\
\text { Global }\end{array}$} \\
\hline & 8,3 & $(1,6)$ & 8 & $(2,1)$ & 8,5 & $(2,2)$ & 7,6 & $(2,6)$ & $7,8 \quad(2,6)$ & 8,3 & & 7 & $(2,7)$ & 7,9 & $(2,1)$ \\
\hline$B(n=11)$ & 7,7 & $(0,9)$ & 8,1 & $(1,2)$ & 7,4 & $(1,7)$ & 5,4 & $(1,8)$ & $6,9 \quad$ (1) & 6 & $(1,3)$ & 5,7 & (1) & 6,7 & \\
\hline$C(n=13)$ & 8,2 & $(1,7)$ & 8,3 & (2) & 7,2 & (2) & 5,3 & $(2,1)$ & $6,6 \quad(1,6)$ & 7,1 & $(1,9)$ & 5,1 & $(1,4)$ & 6,9 & $(1,5)$ \\
\hline$D(n=9)$ & 8 & (1) & 7,8 & $(1,3)$ & 6,6 & $(1,7)$ & 5 & $(1,5)$ & $6,4 \quad(1,7)$ & 6,3 & $(1,6)$ & 4,8 & $(1,9)$ & 6,2 & $(1,3)$ \\
\hline$E(n=10)$ & 7,9 & $(1,2)$ & 6,8 & $(2,2)$ & 5,6 & $(2,1)$ & 4,5 & $(1,8)$ & $6,1 \quad(1,9)$ & 4,8 & $(1,4)$ & 4 & $(1,4)$ & 5,6 & $(1,3)$ \\
\hline$F(n=11)$ & 6,6 & $(1,6)$ & 6,5 & $(1,8)$ & 5 & (1) & 4,1 & $(1,8)$ & 5,6 & 5,9 & $(1,5)$ & 4,9 & $(1,8)$ & 5,3 & $(1,2)$ \\
\hline$G(n=15)$ & 6,8 & $(1,5)$ & 5,7 & $(2,3)$ & 5 & $(1,9)$ & 4,4 & $(2,1)$ & $5,7 \quad(2,2)$ & 5,1 & $(2,4)$ & 4,3 & $(2,3)$ & 5,3 & $(1,8)$ \\
\hline$H(n=15)$ & 6,3 & $(1,5)$ & 5,7 & $(1,9)$ & 4,7 & $(0,9)$ & 4 & $(1,6)$ & $5,2 \quad(1,1)$ & 4,9 & $(1,8)$ & 4,2 & $(1,5)$ & 4,9 & \\
\hline I $(n=5)$ & 5,8 & $(0,6)$ & 4,2 & $(1,6)$ & 5 & $(1,1)$ & 4,3 & $(1,5)$ & $5,2 \quad(1,6)$ & 5,3 & $(1,4)$ & 3,6 & $(2,4)$ & 4,7 & $(1,2)$ \\
\hline Total $(n=94)$ & 7,2 & $(1,5)$ & 6,8 & $(2,2)$ & 5,8 & (2) & 4,8 & (2) & $6,1 \quad(1,8)$ & 5,8 & $(1,9)$ & 4,7 & $(1,9)$ & 5,9 & $(1,5)$ \\
\hline
\end{tabular}




\begin{tabular}{|c|c|c|c|c|c|c|}
\hline $\begin{array}{l}\text { Centro } \\
\text { de Salud } \\
\text { Familiar }\end{array}$ & $\begin{array}{l}\text { Población } \\
\text { total } \\
\text { hipertensa, } \\
\text { según pre- } \\
\text { valencia }\end{array}$ & $\begin{array}{l}\text { Población } \\
\text { total diabé- } \\
\text { tica según } \\
\text { prevalencia }\end{array}$ & $\begin{array}{c}\mathrm{N}^{\circ} \mathrm{de} \\
\text { personas } \\
\text { con } \mathrm{PA}< \\
140 / 90 \mathrm{~mm} \\
\mathrm{Hg}\end{array}$ & $\begin{array}{l}N^{\circ} \text { de perso- } \\
\text { nas con } \\
\text { HbA1c }<7 \%\end{array}$ & $\begin{array}{l}\text { Porcentaje de } \\
\text { Compensación } \\
\text { Hipertensión } \\
\text { arterial }\end{array}$ & $\begin{array}{c}\text { Porcentaje de } \\
\text { Compensación } \\
\\
\text { Diabetes } \\
\text { mellitus } 2\end{array}$ \\
\hline A & 3572 & 2003 & 1263 & 324 & 35 & 16,2 \\
\hline B & 5489 & 2941 & 2532 & 787 & 46 & 27 \\
\hline C & 5785 & 3036 & 2042 & 974 & 35 & 32 \\
\hline D & 2914 & 1606 & 1530 & 372 & 53 & 23 \\
\hline$E$ & 5962 & 3122 & 2710 & 855 & 45 & 27 \\
\hline$F$ & 4162 & 2383 & 1467 & 365 & 35 & 15 \\
\hline G & 5830 & 3170 & 1891 & 596 & 32 & 18,8 \\
\hline $\mathrm{H}$ & 3989 & 2111 & 1977 & 408 & 50 & 19 \\
\hline I & 5752 & 3032 & 1789 & 277 & 31 & 9,2 \\
\hline
\end{tabular}

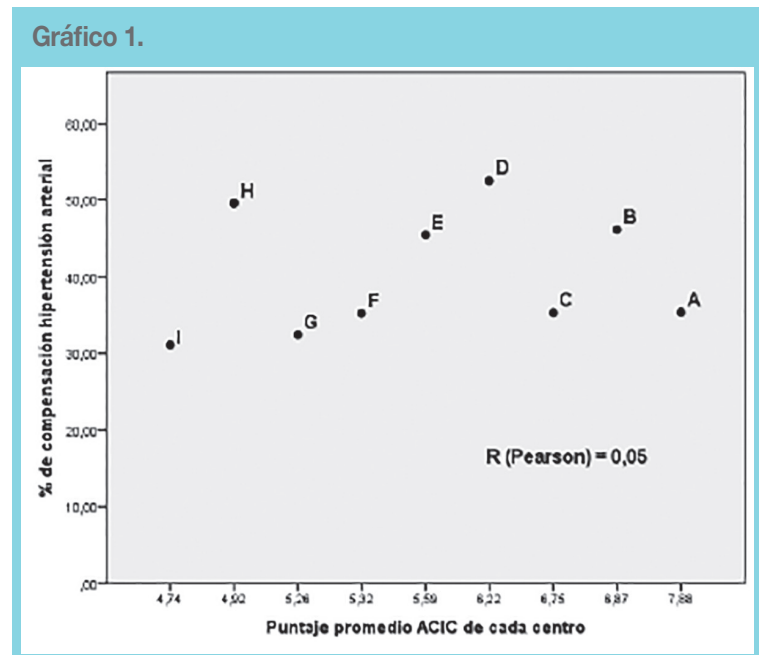

Correlación puntaje promedio de la evaluación de la atención proporcionada a los usuarios/as con enfermedad crónica cardiovascular de cada equipo de salud con porcentaje de compensación de hipertensión arterial.

Por otra parte, los equipos de salud de los Centros representados por las letras A, B, C y D evaluaron la atención otorgada, en forma global, como apoyo razonablemente bueno a la atención de enfermedades crónicas, mientras que el resto de los equipos la evaluó como apoyo básico. La Tabla 4 muestra la distribución numérica y porcentual de los indicadores de compensación para diabetes mellitus tipo 2 e hipertensión arterial de los diferentes Centros de Salud Familiar que componen las comunas del Servicio de Salud.

La Figura 1 ilustra que no hubo una correlación $(\mathrm{R}=0,05)$ entre la variable evaluación de la atención proporcionada

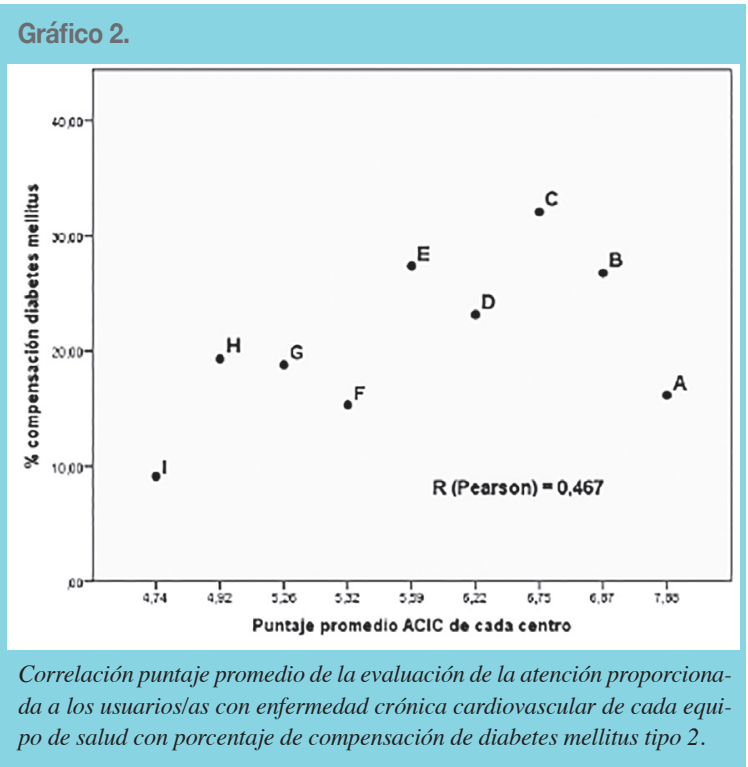

a los usuarios/as con enfermedad crónica cardiovascular y la variable de compensación para la hipertensión arterial. En cambio la Figura 2 ilustra una correlación con tendencia positiva $(R=0,0467)$ entre la variable evaluación de la atención proporcionada a los usuarios/as con enfermedad crónica cardiovascular y la variable de compensación para la diabetes mellitus tipo 2 .

\section{Discusión}

Los resultados extraídos de este estudio han permitido, en primer término, examinar el comportamiento de la implementación del Modelo de Cuidados Crónicos en los establecimientos de atención primaria, así como analizar cómo estos resultados se condicen con el MCC y con estudios previos.

Los equipos del Servicio de Salud estudiados evaluaron la atención otorgada en forma global como apoyo básico a la atención de enfermedades crónicas. Ello concuerda con los resultados reportados por autores en Estados Unidos y en México, al describir las pruebas iniciales en la evaluación global ${ }^{14,16,17}$ y con las orientaciones del propio cuestionario, que señalan que es relativamente común que los equipos de salud que comienzan a trabajar con el MCC obtengan un puntaje bajo 5 en algunas, o todas, las áreas. Estos resultados difieren de los obtenidos en Chile por Arteaga et al, en que la mayoría de los puntajes se ubicaron en un nivel bueno.

Con respecto a los puntajes promedios obtenidos en cada una de las secciones del cuestionario, los equipos coinciden en que la sección mejor evaluada es "Organización del Sistema de atención de salud", equivalente a un apoyo 
razonablemente bueno a enfermedades crónicas. Estos resultados muestran que existe sintonía con el estudio de validación del instrumento ACIC 3.5 realizado en Chile, en que esta sección también fue evaluada como apoyo razonablemente bueno a las enfermedades crónicas. Ello también coincide con resultados obtenidos en investigaciones realizadas en Estados Unidos y Suiza ${ }^{16-18}$. Ello puede deberse a que este componente es una parte importante del modelo que se refiere a la utilización de liderazgo y la oferta de incentivos para mejorar la calidad de la atención ${ }^{19}$. En Chile, en particular, el mayor puntaje obtenido en la evaluación en esta sección puede responder a una serie de esfuerzos que se han realizado para la prevención y control de las enfermedades no transmisibles y la consolidación de las Políticas públicas destinadas a mejorar las enfermedades crónicas ${ }^{11}$.

La segunda sección mejor evaluada correspondió a "Vínculos con la Comunidad", también categorizada como apoyo razonablemente bueno a enfermedades crónicas. Estos resultados son concordantes con los encontrados por otros estudios ${ }^{16-18}$. Ello podría deberse a que esta sección incluye el sistema de salud descrito anteriormente, como también las familias y los hogares, los empleadores, las organizaciones religiosas, el entorno físico y social, los distintos tipos de organizaciones comunitarias y los servicios sociales y educativos, entre otros actores ${ }^{19}$. En Chile, esta buena evaluación podría reflejar los esfuerzos del país en incorporar recursos e innovación a la atención primaria, desde la atención con enfoque de salud familiar, como el trabajo en red vinculado con las comunidades, Consejos de Desarrollo, Comités de Salud locales grupos de autoayuda, entre otros ${ }^{11}$.

La sección "Diseño del Sistema de Atención" fue considerada, en forma general, como apoyo razonablemente bueno a las enfermedades crónicas. Estudios realizados en Holanda, Suiza y Brasil, comparten en que esta sección se encuentra apoyada razonablemente bien ${ }^{18,20,21}$. Esta sección involucra la necesidad de una gestión eficaz de la atención de las enfermedades crónicas, que implica cambios en la organización del sistema para realinear la prestación de atención ${ }^{21}$. No obstante, la mitad de los equipos consideró esta área con un desarrollo básico, lo cual se condice con estudios realizados en Estados Unidos, México y Chile ${ }^{14,16,17}$. Es así como se revela la necesidad de mejorar concretamente en lo que respecta a los componentes sistema de citación de pacientes crónicos, vistas domiciliarias para la atención de enfermedades crónicas, continuidad de la atención, seguimiento de los pacientes en los diferentes niveles de atención de salud y estratificación de riesgo, entre otros. De acuerdo con ello, es imprescindible capacitar a los equipos de salud para que asuman el rol y liderazgo necesario para enfrentar las enfermedades crónicas, anticipándose a los problemas y realizando seguimiento para que el paciente reciba un cuidado efectivo.

La sección "apoyo al autocuidado", fue evaluada como apoyo básico a las enfermedades crónicas. Ello puede deberse a que los equipos consideran que el soporte al autocuidado proporcionado a los usuarios/as está débilmente desplegado, así como también creen que el apoyo a la resolución de inquietudes de los pacientes y familiares se encuentra solo básicamente desarrollado. También responden a que falta desarrollar intervenciones efectivas para el cambio de conducta y el apoyo de pares. Resultados similares se encontraron en estudios realizados en Chile, Holanda y Suiza ${ }^{18,20}$.

La sección "Sistema de Información Clínica" fue evaluada como apoyo básico a las enfermedades crónicas. Este resultado se debe principalmente a que los equipos consideran que falta desarrollo de la implementación de listas o directorios de pacientes con patologías específicas; asimismo, es necesario mejorar la retroalimentación entre los equipos de salud. Lo anterior es inquietante, ya que en Chile se han experimentado mejoras para disponer de tecnologías de información que permitan identificar a la población en riesgo, así como también organizar la atención, monitorear la respuesta al tratamiento y evaluar los resultados. Sin embargo, estos resultados son similares a los obtenidos en un estudio realizado en Brasil ${ }^{21}$, en donde esta sección fue la más débil. Los sistemas de información clínica organizan la información sobre pacientes individuales y poblaciones para ayudar a identificar necesidades, planificar la atención en el tiempo, dar seguimiento a las respuestas al tratamiento y evaluar los resultados de salud. Por ello, son el corazón de un Modelo efectivo de Cuidados Crónicos ${ }^{19}$.

Por último, las secciones con más bajos puntajes, fueron "Apoyo a la toma de decisiones" e "Integración de los componentes del modelo" y se categorizaron como apoyo básico a las enfermedades crónicas. Respecto de la sección “Apoyo a la Toma de Decisiones", los resultados encontrados pueden deberse principalmente a que los equipos de salud consideran básicamente desarrollada la participación de los especialistas en el mejoramiento de la atención, la capacitación de los equipos de atención a enfermedades crónicas, las guías clínicas y la información a los usuarios/as con enfermedad crónica. De este modo, esta área se convierte en una de las más débiles y que requiere de 
fortalecimiento. Para ello es preciso incorporar más especialistas, capacitar respecto de la mejor forma de atención y aplicar guías de práctica clínica con protocolos integrados y basados en la evidencia. También se requieren equipos de trabajo que cuenten con la colaboración de otras disciplinas.

En relación a la sección "Integración de los Componentes del Modelo", los hallazgos encontrados orientan a que, al momento de planificar mejoras, se consideren no solo aquello aspectos débiles o básicos, sino todos los aspectos del Modelo de Cuidados Crónicos, con el propósito de mejorar resultados y controlar costos ${ }^{10,20}$. Lo señalado se sustenta en las sugerencias propuestas en estudios preliminares de diversos países que han levantado información respecto de la forma de otorgar cuidados a usuarios/as con enfermedad crónica, así como también en la proyección de intervenciones para optimizar la atención proporcionada ${ }^{16-23}$.

Con respecto a los indicadores de compensación para la diabetes mellitus tipo 2 y para la hipertensión arterial, se pudo observar que hubo Centros de Salud Familiar que se ubicaron bajo la meta establecida para lograr una cobertura efectiva en diabetes mellitus tipo 2 , según prevalencia y antecedentes epidemiológicos ${ }^{\mathfrak{f}}$. Sin embargo, el resto del establecimiento dio cumplimiento a lo programado para esta afección. Con respecto a la hipertensión arterial se observó que sólo la mitad de los Centros de Salud Familiar alcanzaron a dar cumplimiento a la meta propuesta, dejando de manifiesto la necesidad de mejorar la cobertura efectiva de la hipertensión arterial en este Servicio de Salud. Ello da cuenta de que, si bien se ha aumentado el número de personas bajo control compensadas, todavía este porcentaje es insuficiente ${ }^{24}$. Es imprescindible tomar medidas, ya que el cumplimiento de los indicadores de compensación es insuficiente para el cumplimiento con los objetivos sanitarios propuestos para el 2011-2020, (estrategia $\mathrm{N}^{\circ} 2$, respecto del incremento de la proporción de personas con diabetes mellitus e hipertensión arterial controlada y del Plan de acción mundial sobre las Enfermedades No Transmisibles ${ }^{25}$ ).

Con relación a los equipos, es posible identificar que los centros A, B, C y D se caracterizan por evaluar la atención otorgada como apoyo razonablemente bueno y por presentar al menos un indicador de compensación (diabetes mellitus y/o hipertensión arterial) sobre la meta comunal, a diferencia del resto de los centros que evaluó la atención como apoyo básico y presentó sólo un indicador de compensación (diabetes mellitus o hipertensión arterial) sobre la meta comunal.
Respecto de la relación entre la evaluación de la atención proporcionada por los equipos de salud a usuarios/as con enfermedad crónica cardiovascular y los indicadores de compensación para diabetes mellitus e hipertensión arterial, se encontró una correlación solo con tendencia positiva, para diabetes mellitus. Un estudio realizado en Australia, reportó una relación estadística entre la evaluación de la atención proporcionada, medida a través del ACIC, con la calidad de la atención en diabetes ${ }^{26}$. Otros estudios con participación de usuarios diabéticos han reportado una relación significativa entre la puntuación ACIC y la hemoglobina A1c, mediada por el autocuidado ${ }^{27}$ y también entre los componentes de MCC y los factores de riesgo para enfermedad cardiovascular ${ }^{28}$. La diferencia con los hallazgos del presente estudio puede deberse a que en los estudios citados la variable respuesta fue estudiada de forma individual y no como variable resumen a nivel de los centros de salud.

Estos resultados sugieren incorporar en futuras investigaciones otras variables relacionadas con la calidad de la atención como edad, sexo, la percepción de los usuarios/ as con enfermedad crónica, autocuidado, forma de control de los parámetros de compensación y capacitación de los equipos, entre otras ${ }^{23}$.

En cuanto a limitaciones, cabe mencionar que no fue posible obtener los datos de un número importante de integrantes de los equipos de salud cardiovascular, generando un posible sesgo de selección ${ }^{29}$. Algunas de las razones que impidieron su participación fueron: falta de tiempo de los profesionales para responder el cuestionario, profesionales con licencia médica durante el periodo de recolección de datos, desvinculación en algunos Centros de Salud durante el periodo de estudio y paralizaciones de tipo gremial. Por lo tanto, los datos se obtuvieron de una muestra relativamente pequeña, lo que significa que otros equipos de comunidades rurales o situados en otras regiones pueden mostrar un patrón diferente de resultados. En conclusión, la atención proporcionada a los usuarios/as con enfermedad crónica cardiovascular fue evaluada como apoyo básico a las enfermedades crónicas, sin encontrarse relación estadísticamente significativa con los indicadores de compensación para diabetes mellitus tipo 2 e hipertensión arterial.

Agradecimientos: Las/os autoras/es agradecen el patrocinio otorgado por el Servicio de Salud en donde se realizó el estudio y al Dr. Oscar Arteaga, por facilitar la versión adaptada al Castellano Chileno del Cuestionario Assessment of Chronic Illness Care (ACIC-EVACE).

$£$ Metas de compensación por comuna. Números romanos (I al IV) representan a cada comuna. I: CESFAM A, B, I; II: CESFAM G; III: CESFAM C, D, F, J; IV: CESFAM E, H. Meta de compensación para diabetes mellitus tipo 2: I $26 \%$; II $18,8 \%$; III $22,4 \%$ y IV 19,4\%. Meta de compensación para hipertensión arterial: I 35\%; II 39,4\%; III 41,7\% y IV 48.8\%. DEIS. MINSAL. MANUAL DE INSTRUCCIONES REM 2013. SERIE P. 


\section{Referencias}

1. CHOW CK, TEO KK, RANGARAJAN S, ISLAM S, GUPTA R, AVEZUM A, et al. Prevalence, awareness, treatment, and control of hypertension in rural and urban communities in high-, middle-, and low-income countries. JAMA. 2013; 310:959-68.

2. CHACÓN J, SANDOVAL D, MUÑOZ R, ROMERO T. Evaluación del control de la presión arterial y la adherencia terapéutica en hipertensos seguidos en el Programa de Salud Cardiovascular (PSCV). Asociación con características clínicas, socioeconómicas y psicosociales. Rev Chil Cardiol. 2015; 34: 18-27.

3. SEREMI DE SALUD REGIÓN DEL BIOBÍO. Atlas de salud 204 Región del Bío-Bío [Internet]. Concepción: Departamento de Salud Pública y Planificación Sanitaria; 2014 [citado 20 julio 2016]. 102 p. Disponible en: www.seremidesaludbiobio.cl/epidemiologia/archivos/2014/AtlasSalud.pdf

4. ORGANIZACIÓN PANAMERICANA DE LA SALUD. CONTROL DE LA DIABETES [Internet]. Washington, DC: OPS; 2015 [citado 17 agosto 2016]. Disponible en: http://www.paho. org/world-health-day/?page_id=7972\&lang=es

5. ORGANIZACIÓN MUNDIAL DE LA SALUD. Preparación de los profesionales de la atención de salud para el siglo XXI: el reto de las enfermedades crónicas [Internet]. Ginebra: OMS; 2005 [citado 30 marzo 2015]. 73 p. Disponible en: http://whqlibdoc.who.int/publications/2005/9243562800_spa.pdf

6. MINISTERIO DE SALUD DE CHILE. Modelo de Atención Integral en Salud. Serie Cuadernos Modelo de Atención $\mathrm{N}^{\circ} 1$ [Internet]. Santiago, Chile: Subsecretaria de Redes Asistenciales/ División de Gestión de la Red Asistencial/MINSAL; 2005. [citado 17 julio 2015]. 27 p. Disponible en: http:// www.minsal.gob

7. ORGANIZACIÓN MUNDIAL DE LA SALUD/PUBLIC HEALTH AGENCY OF CANADA. Prevención de las enfermedades crónicas: una inversión vital [Internet]. Ginebra: OMS; 2005. [citado 12 junio 2015]. 30 p. Disponible en: http:// www.who.int/chp/chronic_disease_report/en/

8. CONSEJO INTERNACIONAL DE ENFERMERAS. Servir a la Comunidad y garantizar la calidad: Las enfermeras a la vanguardia de los cuidados de las enfermedades crónicas [Internet]. Ginebra: Consejo Internacional de Enfermeras; 2010 [citado 17 julio 2015]. 75 p. Disponible en: http://www.icn.ch/es/publications/2010-delivering-quality-serving-communities-nurses-leading-chronic-care/

9. CONSEJO INTERNACIONAL DE ENFERMERAS. Uníos a la lucha contra las enfermedades crónicas [Internet]. Ginebra: Consejo Internacional de Enfermeras; 2010. [citado 20 julio
2015]. Disponible en: http://www.icn.ch/es/news/icn-press-releases-2010/comunicado-de-prensa-del-cie-2010-683.html

10. MINISTERIO DE SALUD. METAS 2011-2020. Elige vivir sano. Estrategia nacional de salud para el cumplimiento de los objetivos sanitarios para la década 2011-2020: [Internet]. Santiago, Chile: MINSAL; 2011 [citado 25 agosto 2015]. 347 p. Disponible en: http:// www.minsal.cl.portal/url/item/c4034eddbc96ca6de0400101640159b8.pdf

11. MINISTERIO DE SALUD. Manual de apoyo a la implementación del Modelo de Atención Integral de Salud con enfoque Familiar y Comunitario en establecimientos de Atención Primaria Subsecretaría de Redes Asistenciales División de Atención Primaria Departamento de Diseño y Gestión de APS. [Internet]. 2008. [citado 25 agosto 2015]. Disponible en: http:// www.minsal.cl.

12. WAGNER EH, AUSTIN BT, DAVIS C, HINDMARSH M, SCHAEFER J, BONOMI A. IMPROVING CHRONIC ILLNESS CARE: Translating Evidence Into Action. Health Affairs. 2001; 20:64-78.

13. WAGNER EH, SANDHU N, COLEMAN K, PHILLIPS KE, SUGARMAN JR. Improving care coordination in primary care. Med Care. 2014; 52:S33-8.

14. BONOMI AE, WAGNER EH, GLASGOW RE, VON KORFF M. Assessment of Chronic Illness Care (ACIC): A Practical Tool to Measure Quality Improvement. Health Serv Res. 2002; 37:791-820

15. RODRÍGUEZ E. Comités de Evaluación Ética y Científica para la Investigación en Seres Humanos, propuestos por Ezekiel Emanuel. Siente Requisitos Éticos. Acta Bioética. 2004; 10: 37-48.

16. SOLBERG LI, CRAIN AL, SPERL-HILLEN JM, HROSCIKOSKI MC, ENGEBRETSON KI, O'CONNOR PJ. Care Quality and Implementation of the Chronic Care Model: A Quantitative Study. Ann Fam Med. 2006; 4:310-6.

17. BARCELÓ A, CAFIERO E, DE BOER M, MESA AE, LOPEZ MG, JIMÉNEZ RA, et al. Using collaborative learning to improve diabetes care and outcomes: the VIDA project. Prim Care Diabetes. 2010;4:145-53.

18. CRAMM JM, STRATING MM, TSIACHRISTAS A, NIEBOER AP. Development and validation of a short version of the Assessment of Chronic Illness Care (ACIC) in Dutch Disease Management Programs. Health Qual Life Outcomes [Internet]. 2011 [cited 17 agosto 2016]; 9:49. Disponible en: http://www. 
ncbi.nlm.nih.gov/pubmed/21726439

19. ORGANIZACIÓN PANAMERICANA DE LA SALUD. Cuidados innovadores para las condiciones crónicas: Organización y prestación de atención de alta calidad a las enfermedades crónicas no transmisibles en las Américas. Washington, DC: OPS; 2013.

20. STEURER-STEY C, FREI A, SCHMID-MOHLER G, MALCOLM-KOHLER S, ZOLLER M, ROSEMANN T. THE GERMAN version of the Assessment of Chronic Illness Care: instrument translation and cultural adaptation. J Eval Clin Pract. 2012;18:1-4.

21. COSTA KC, CAZOLA LHO, TAMAKI EM. Assessment of Chronic Illness Care (ACIC): avaliação da aplicabilidade e resultados. Saúde debate. 2016; 40:106-117.

22. TSAI AC, MORTON SC, MANGIONE CM, KEELER EB. A Meta-Analysis of Interventions to Improve Care for Chronic Illnesses. Am J Manag Care. 2005; 11: 478-488.

23. FREI A, SENN O, CHMIEL C, REISSNER J, HELD U, ROSEMANN T. Implementation of the chronic care model in small medical practices improves cardiovascular risk but not glycemic control Diabetes Care. 2014; 37: 1039-47.

24. GOBIERNO DE CHILE. Subsecretaria de salud pública división prevención y control de enfermedades. Dpto. Enfermeda- des no transmisibles implementación del enfoque de riesgo en el programa de salud cardiovascular. [Internet]. 2009 [citado 25 abril 2015]. Disponible en: http://www.minsal.cl. /portal/ur1/787e4765248bc9e0e04001011f0172b5.pdf.

25. ORGANIZACIÓN MUNDIAL DE LA SALUD. Informe sobre la situación mundial de las enfermedades no transmisibles 2014. Ginebra: OMS. [Internet] 2014. [citado 12 octubre 2015]. Disponible en: http://www.who.int/nmh/publications/ncd-status-report-2014/es/

26. SI D, BAILIE R, CONNORS C, DOWDEN M, STEWART A, ROBINSON G, et al. Assessing health centre systems for guiding improvement in diabetes care. BMC Health Serv Res [Internet]. 2005 [cited 17 agosto 2016]; 5:56. Disponible en: http:// www.ncbi.nlm.nih.gov/pmc/articles/PMC1208882/

27. PARCHMAN ML, PUGH JA, WANG CP, ROMERO RL. Glucose control, self-care behaviors, and the presence of the chronic care model in primary care clinics. Diabetes Care. 2007; 30:2849-54.

28. PARCHMAN M, KAISSI AA. Are elements of the chronic care model associated with cardiovascular risk factor control in type 2 diabetes? Jt Comm J Qual Patient Saf. 2009;35:133-8.

29. HERNÁNDEZ B, VELASCO-MONDRAGÓN HE. Encuestas transversales. Salud pública Méx. 2000;42: 447-455. 